\title{
A new sledge jump system that allows almost natural reactive jumps
}

\author{
A. Kramer ${ }^{a}, *$ R. Ritzmann ${ }^{a}$, A. Gollhofer ${ }^{a}$, D. Gehring ${ }^{a}$, M. Gruber ${ }^{b}$ \\ Department of Sport and Sport Science, University of Freiburg, Germany \\ beportment of Training- and Movement Science, University of Potsdam, Germany
}

A RTICLE IN FO

Keywords:

Sledge

SSC

Motion analysis

Hopping

Electromyography

\begin{abstract}
A B S T R A C T
Aim: Sledge jump systems (SIS) are often employed to examine the underlying mechanical and neuromuscular mechanisms of the stretch-shortening cycle (SSC) as they allow the systematic variation of impact velocity and energy. However, in existing SIS the jumps are not very comparable to natural jumps because of the long contact times $(\sim 200 \%)$, which prevent the storage of kinetic energy. The aim of the present study was to evaluate if an ultra-light sledge, built in a way that joint movement is barely restricted, allows jumps that are comparable to natural jumps.

Methods: Ground reaction forces, kinematic and electromyographic (EMG) data of 21 healthy subjects were compared between normal hoppings (NH) on the ground and hoppings in a custom-built SJS (sledge hoppings, $\mathrm{SH}$ ).

Results: Normalized to $\mathrm{NH}$, the ground contact times for the SH were prolonged $(+22 \%)$, while the peak forces $(-21 \%)$ and the preactivity of the soleus and gastrocnemius medialis muscles were reduced $(-20 \%$ and $-22 \%$, respectively). No significant changes were observed for the iEMG of the short latency response of those muscles $(+1 \%$ and $+8 \%$ ) and the ranges of motion in the ankle, knee and hip joint (differences of 1,1 and 2 degrees). The reduced peak forces were associated with a reduced leg stiffness $(-21 \%)$.

Conclusion: The new system allows reactive jumps that are rather comparable to natural jumps. Therefore, the new SJS seems to be an adequate system in order to examine the SSC under controlled and almost natural conditions.
\end{abstract}

\section{Introduction}

Sledge jump systems (SJS) are a common methodological approach to study the complex nature of the stretch-shortening cycle (Bubeck and Gollhofer, 2001; Komi et al., 1987). The stretchshortening cycle (SSC) can be defined as the stretching of a preactivated muscle-tendon-unit (MTU), immediately followed by a contraction of the muscle (Komi, 1984). The advantage of this type of muscle action compared to a purely concentric one is the higher efficiency, caused by a storage of energy in the elastic elements in the eccentric phase and its release in the following concentric phase (Asmussen and Bonde-Petersen, 1974; Fukashiro et al., 2006; Gollhofer, 1987; Henchoz et al., 2006). During locomotion, especially when running or jumping, most mammals (including man) take advantage of the energy storage in the SSC. Since energy-efficient movements are important for everyday activities as well as for many sports, the SSC has been studied

\footnotetext{
* Correspondence to. IfSS der Albert-Ludwigs-Universität Freiburg. Schwarzwaldstraße 175, 79117 Freiburg. Germany. Tel.: +49761 2034557 ; fax: +497612034534 .

E-mail addresses: andieas.kramer@sport.uni-freiburg.de. andreas_kramer@web.de (A. Kramer).
}

extensively (Bosco et al., 1982; Finni et al., 2001; Horita et al., 2003; Komi and Gollhofer, 1997).

SJSs allow a detailed examination of the neuromuscular control during the different phases of the SSC because they allow the controlled variation of important parameters such as acceleration, impact velocity and energy without changing the jump height (and thus the stiffness, the timing and the motor program). For example, the variation of the impact energy (achieved either by altering the mass of the system or the velocity) has been used to assess the influence of the stretch load and velocity on the behavior of the neuromuscular system during the eccentric and concentric phases of the jump (Kyröläinen and Komi, 1995). Furthermore, the acceleration in a SJS is not predetermined by gravity (as it is the case for normal jumps) but can be set within a wide range, allowing the examination of unloading effects and the simulation of different gravity conditions (Bubeck, 2002). Another methodological advantage of SJSs is that equipment that would normally affect the participant during an experiment (like a coil for transcranial magnetic stimulation of the motor cortex) can be attached to the sledge instead of the participant, thus eliminating or at least reducing adverse effects on the recordings.

The technical approaches that were used to build such SJSs are diverse: the research group of Komi designed a sledge on which the subject sits and rolls down on two rails with adjustable inclination 
(Komi et al., 1987). Bubeck and Gollhofer (2001) used a horizontal sledge upon which the subject lies and is pulled towards a vertical plate by a rack of weights. But none of those systems were able to provide natural jumping conditions: the ground contact times were very long (twice as long compared to natural jumps) and the preactivity of the leg muscles as well as the peak ground reaction forces and the leg stiffness were low (less than 50\% compared to natural jumps) (Bubeck and Gollhofer, 2001). It has been suggested that the reason for this is the high mass of the sledge's moving parts and the lacking freedom of movement in the involved joints (Bubeck and Gollhofer, 2001). Consequently, the jumps lack essential characteristics of the SSC which prevent or at least greatly reduce the storage of kinetic energy in the MTU.

The aim of the present study was to evaluate if jumps in a new SJS with an ultra-light sledge - built in a way that movement of the ankle, knee and hip joints is barely restricted - fulfilled the following criteria: short ground contact times, high peak ground reaction forces, high preactivity of the leg extensor muscles and a range of motion of the joint angles similar to those of natural jumps. For this purpose, kinetic, kinematic and electromyographic parameters of normal bilateral hoppings on the ground were compared to those of sledge hoppings. Hoppings were chosen because they possess all the characteristics of reactive jumps using the SSC, making them representative, and because they allow a high number of repetitions, which makes them more reliable than for example drop jumps. Other kinds of jumps were not included as it was not the aim of the study to test if specific types of jumps were possible, but rather to evaluate if any kind of stiff, reactive jump was possible in a SIS, something that could not be achieved with previous systems.

\section{Methods}

\subsection{Subjects}

Twenty-one healthy subjects ( 9 females, 12 males) volunteered to participate in this study. The participants were physically active students at the deparinent of sports science. All participants gave written informed consent to the experimental procedure, which was approved by the ethics committee of the University of Freiburg and in accordance with the latest revision of the Declaration of Helsinki. All participants were healthy with no previous neurological irregularities or injuries of the lower extremity. Their mean ( \pm standard deviation, SD) height. weight and age were $176 \pm 10 \mathrm{~cm}, 73 \pm 13 \mathrm{~kg}$ and $24 \pm 4$ years, respectively.

\subsection{Experimental design}

A single-group repeated-measures study design was used to examine differences between normal hoppings (NH) and sledge hoppings ( $\mathrm{SH}$ ) on the basis of the recorded ground reaction forces (GRF), the electromyographic (EMG) activity of six leg muscles and a motion analysis. In addition, normal hoppings were performed with an additional load of $5 \mathrm{~kg}$ (weight vest), in order to mimic the influence of the mass of the sledge: these hoppings will be called loaded hoppings (LH). After a ten-min warm-up phase (consisting of running, tappings and hoppings), the hoppings were performed with the instruction to jump as stiff as possible, i.e. to keep the contact time as short as possible, ensuring more consistent and thus more reliable jumps. One trial consisted of 40 hoppings with a two-min break after the first 20 hoppings to reduce possible effects of fatigue. In between trials, the subjects were given as much time to recover as they deemed necessary. The jumps were performed bare-footed on two force plates (AMTIR, Watertown, USA for the normal jumps with and without additional load; in the SJS, two Leonardo(B) platforms by Novotec Medical GmbH, Pforzheim, Germany were used). The GRF perpendicular to the force plates were recorded separately for the right and the left foot, to ensure that the EMG activity of the right leg would be synchronous with the signal of the right force plate, acting as a trigger signal. The order of the test conditions was balanced between subjects to control confounding effects such as fatigue.

\subsection{Sledge jump system}

The SIS (see Fig. 1) was developed by Novotec Medical GmbH (Pforzheim Germany). It consists of a tilting table and a lightweight sledge ( $5 \mathrm{~kg}$ ) that is attached to a rail on both sides of the table. The tilt angle of the table can be adjusted continuously between zero (horizontal position, used in this study) and 90 degrees (vertical position). The construction allows the sledge only to move alongside the table, i.e. when the table is in a horizontal position, the sledge can slide only in a horizontal direction. Note though that the sledge is attached to the rails with straps that allow some movement perpendicular to the movement direction as well as some rotation (see Fig. 2). Thus, the participant's movement is barely restricted and he can move in a more natural way. He is attached to the sledge via four straps, two around the thighs and two around the shoulders

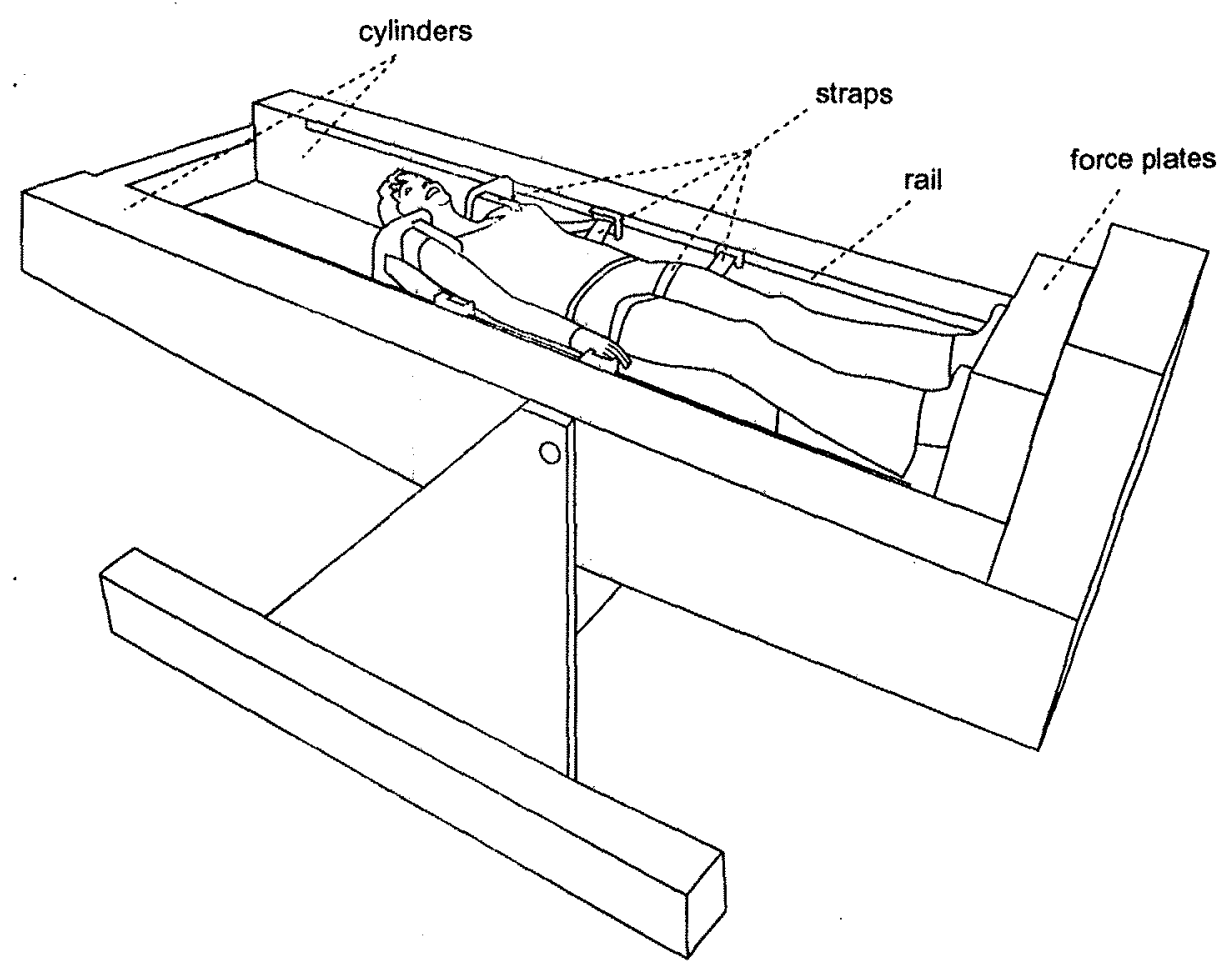

Fig. 1. The sledge jump system in the horizontal position. The participant is fixed to the wooden sledge with straps. The straps are attached to the rails and can slide in the direction of the rails. The participant stands on two force plates (separated, one for each foot). 

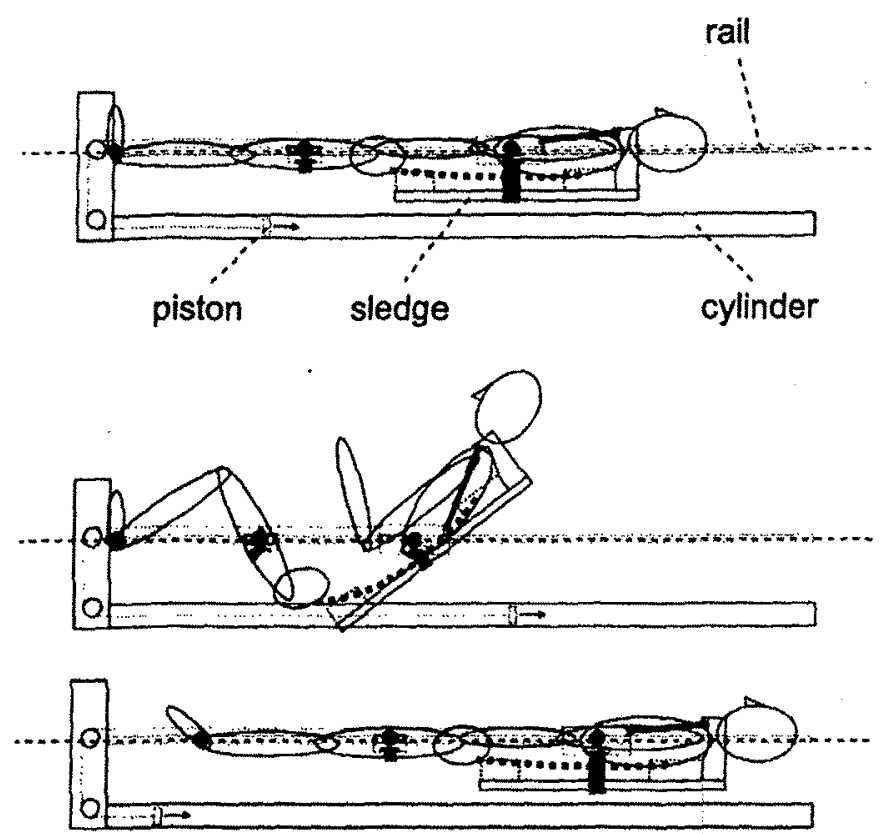

Fig. 2. A longitudinal section of the SJS. The two low-pressure cylinders pull the participant onto the force plates. The second drawing illustrates the freedom of movement in the ankle. knee and hip joint (the downward movement of the hip is not constrained by the cylinders like the drawing might suggest, as the cylinders are to the left and right of the participant, not below: see Fig. 1). Note that the figure is not intended to show a hopping.

(see Fig. 1). The force that pulls the sledge in the movement direction is generated by two low-pressure cylinders. One cylinder working at full capacity generates $500 \mathrm{~N}$, i.e. any force between 0 and $1000 \mathrm{~N}$ can be set by altering the pressure of the cylinders. In this study, the pressure was adjusted in a way that the forces produced by the cylinders matched the subject's weight. The SIS was used in the horizontal position because that way, no gravitational forces act in the direction of the jump. only the forces generated by the cylinders.

\subsection{Kinematics}

The hoppings were recorded with a marker-based motion capturing system (Vicon, Oxford, UK) using ten cameras (MX40,200 Hz). The markers were placed on the following anatomical landmarks of the right leg: hallux, fifth metatarsal bone, lateral malleolus, lateral knee joint center and greater trochanter, in addition, one marker was placed on the sternum. Those markers were used to generate a $2 \mathrm{D}$-model of the right leg. from which three joint angles were calculated (ankle, knee and hip).

\subsection{Electromyography}

Bipolar Ag/AgCl surface electrodes (Ambu Blue Sensor P, Ballerup, Denmark; diameter $9 \mathrm{~mm}$, center-to-center distance $34 \mathrm{~mm}$ ) were placed over $M$. soleus (SOL). M. gastrocnemius medialis (GM). M. tibialis anterior (TA), M. rectus femoris (RF), $M$. vastus lateralis (VL) and $M$. biceps femoris (BF) of the right leg. The longitudinal axes of the electrodes were in line with the presumed direction of the underlying muscle fibers. The reference electrode was placed on the patella Interefectrode resistance was kept below $2 \mathrm{k} \Omega$ by means of shaving, light abrasion and degreasing of the skin with a disinfectant.

The EMG signals were transmitted via shielded cables to the amplifier (bandpass filter $10 \mathrm{~Hz}$ to $1 \mathrm{kHz}, 1000 \times$ amplified) and recorded with $4 \mathrm{kHz}$.

\subsection{Dato processing}

The EMG signals were set to zero to correct possible offsets and then rectified. Afterwards, mean and standard deviation of the EMG and force signals were calculated. using the GRF of the right force plate as a trigger signal. The IEMG was calculated by integrating the mean EMG signal of four time intervals, based on previous reported latencies and durations of the reflex components (Lee and Tatton. 1978: Marsden et al 1978: Sinkjaer et al., 1999): the preactivity phase (PRE) from 150 ms before ground contact (GC) until GC, the phase of the short latency response (SLR) from 30 ms after GC until $60 \mathrm{~ms}$ after $C C$, the phase of the medium latency response (MLR) from $60 \mathrm{~ms}$ after $G C$ until $90 \mathrm{~ms}$ after $G C$ and the phase of the late latency response (LLR) from $90 \mathrm{~ms}$ after GC until $120 \mathrm{~ms}$ after GC (see Fig. 3). The ground contact time (GCT) was determined as the time interval between $\mathrm{C} C$ and takeoff. The rate of force development (RFD) was calculated as the peak force divided by the time from GC until the force signal reached its peak. The joint angles were determined at the time of GC and the range of motion (ROM) was calculated for the GCT. The right leg's stiffness was calculated according to Günther and Blickhan (2002) as the ratio of the peak GRF and the displacement of the hip marker (greater trochanter) during the time interval from $G C$ until the GRF reached its peak.

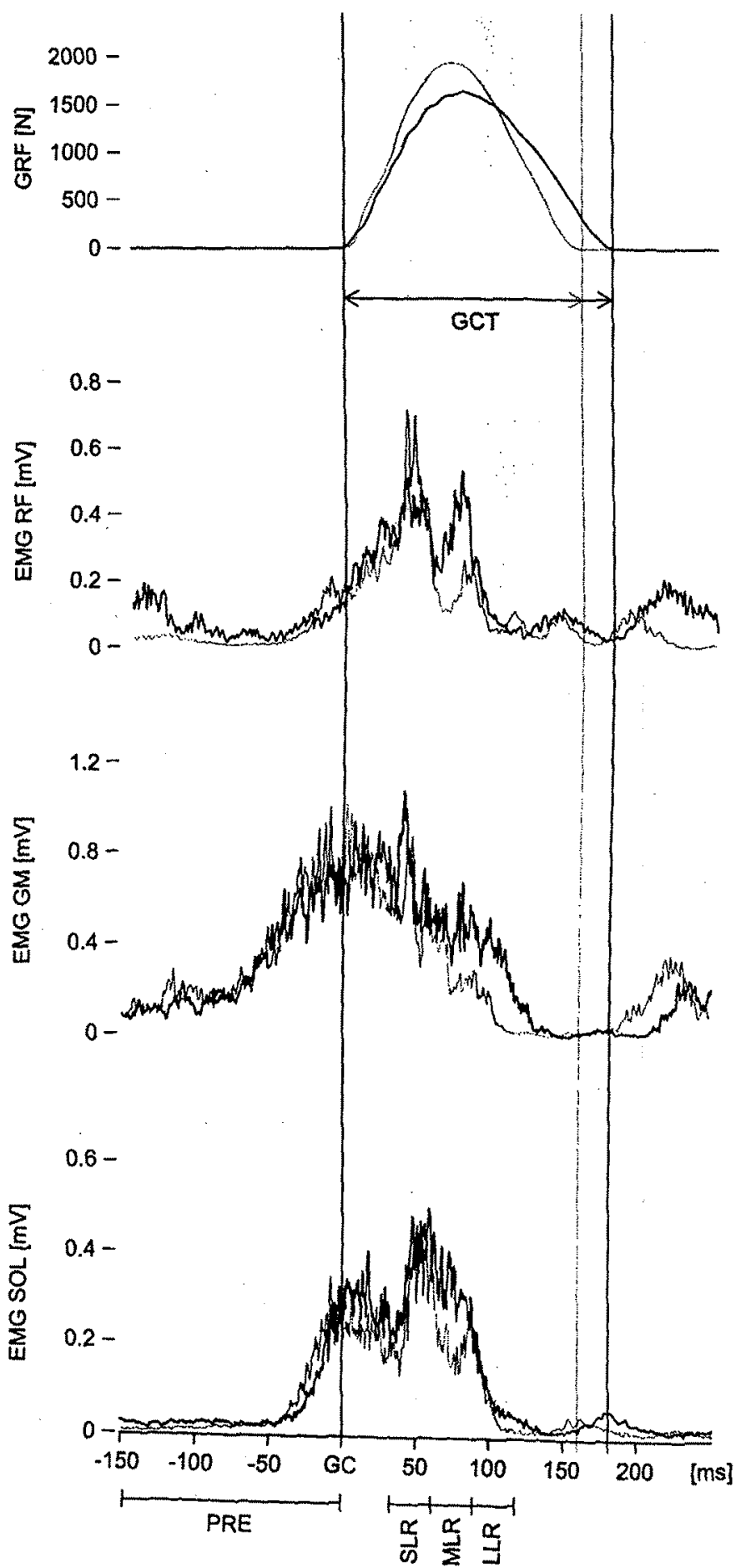

Fig. 3. Averaged force and EMG data of $2 \times 40$ hoppings from one exemplary participant. Black curves represent the sledge hoppings, the grey ones represent the normal hoppings. The ground contact time (CCT) is marked as the time between ground contact and takeoff. Also marked are the relevant EMG phases: PRE $150 \mathrm{~ms}$ before GC until GC. SLR 30-60 ms after GC, MLR 60-90 ms and LLR 90-120 ms. 


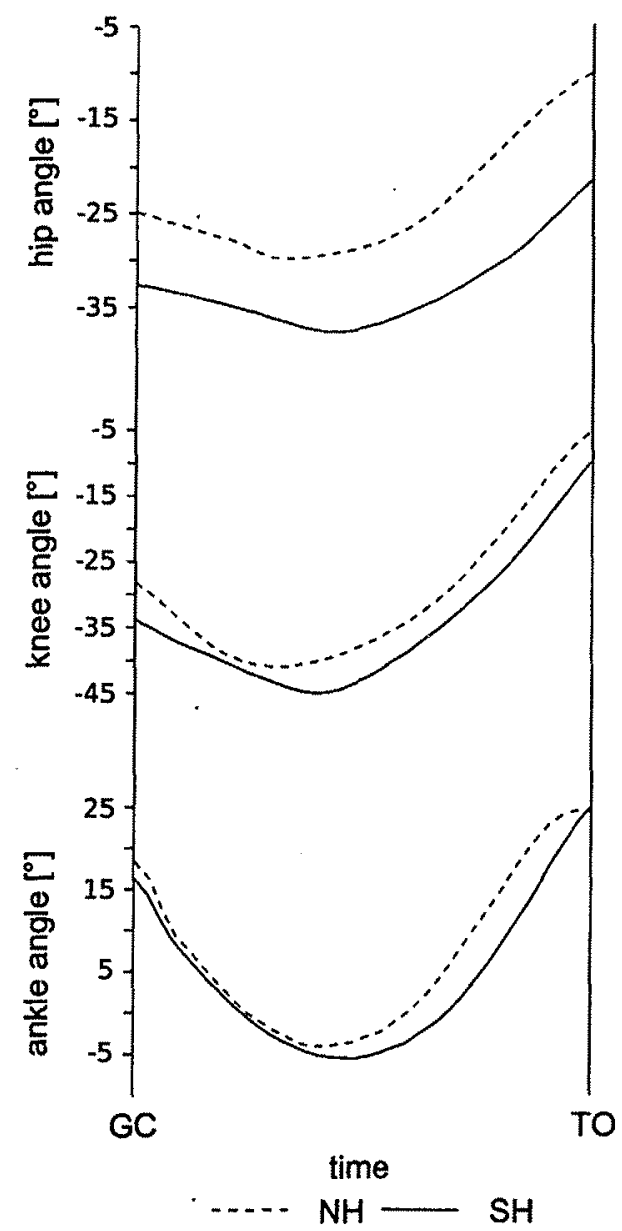

Fig. 4. Mean joint angle progression for one exemplary participant $(2 \times 40$ hoppings), normalized to the ground contact time of the normal hoppings. The progression is quite similar when comparing $\mathrm{SH}$ to $\mathrm{NH}$, only with an offset (most pronounced for the hip), i.e. the joints are generally more flexed during sledge hoppings. Negative values indicate more flexion than during upright stance.

\subsection{Statistics}

To compare the values obtained in the SIS to the ones of the normal condition, the SJS values were divided by (or subtracted from, in case of the joint angles) the values obtained for the NH for each subject and then averaged for all subjects. This descriptive data presentation shows the size of the differences in the recorded parameters, which was the main goal of the present study. In addition equivalence statistics were used to determine if the mean values of the two groups could be considered statistically equal. For this purpose, the $90 \%$ confidence interval ( $\mathrm{Cl}$ ) was calculated for the differences between the two groups. The acceptable bounds were determined for each parameter separately. based on the differences observed between the first 20 and the second 20 jumps of the $\mathrm{NH}$ (Borman et al., 2009). If the differences were statistically insignificant (i.e. if the $\mathrm{Cl}$ lay within those bounds), the respective parameter is marked with a superscript $\approx$ symbol. Group data are presented as means \pm standard deviations (SD) unless otherwise stated.

\section{Results}

\subsection{Forces}

In Table 1, the absolute peak forces, the RFD and the GCT of the $\mathrm{NH}$ are displayed alongside the relative values for the $\mathrm{SH}$. In case of the peak force, the values for both legs are the combined values of the right and left leg. In case of the RFD and the GCT, the averaged values of the right and left leg are displayed. The peak forces for the SH compared to the NH were about $20 \%$ smaller, the GCT $20 \%$ longer and consequently the RFD was approximately $30 \%$
Table 1

Mean values of all participants of the peak ground reaction force (GRF), the average rate of force development (RFD) and the ground contact time (GCT) for the normal hoppings (NH) and the respective relative values for the sledge hoppings (SH) and the loaded hoppings (LH).

\begin{tabular}{lccc}
\hline & Peak force & RFD & GCT \\
\hline NH & $3478 \pm 927 \mathrm{~N}$ & $24.2 \pm 7.2 \mathrm{kN} / \mathrm{s}$ & $168 \pm 15 \mathrm{~ms}$ \\
SH & $79 \pm 9 \%$ & $68 \pm 8 \%$ & $122 \pm 14 \%$ \\
LH & $102 \pm 4 \% \approx$ & $96 \pm 6 \% \approx$ & $107 \pm 5 \%$ \\
\hline
\end{tabular}

Table 2

Mean EMG activity of all participants for the six recorded muscles during four phases (PRE $150 \mathrm{~ms}$ before GC until GC, SLR 30-60 ms after GC, MLR 60-90 ms and LLR $90-120 \mathrm{~ms}$ ). Absolute values in $\mathrm{mV}^{*} \mathrm{~s}$ for the normal hoppings and relative values in percent for the sledge and loaded hoppings.

\begin{tabular}{|c|c|c|c|c|}
\hline & PRE & SLR & MLR & LLR \\
\hline SOL NH (mVs) & $0.013 \pm 0.006$ & $0.011 \pm 0.004$ & $0.010 \pm 0.003$ & $0.004 \pm 0.003$ \\
\hline SH (\%) & $80 \pm 39$ & $101 \pm 25 \approx$ & $105 \pm 37=$ & $182 \pm 128$ \\
\hline$L H(\%)$ & $91 \pm 22 \approx$ & $103 \pm 9$ & $103 \pm 14^{*}$ & $116 \pm 35$ \\
\hline GM NH (mVs) & $0.028 \pm 0.012$ & $0.013 \pm 0.005$ & $0.009 \pm 0.004$ & $0.003 \pm 0.003$ \\
\hline $\mathrm{SH}(\%)$ & $78 \pm 16$ & $108 \pm 25$ & $137 \pm 69$ & $313 \pm 254$ \\
\hline LH (\%) & $97 \pm 18 \approx$ & $99 \pm 15^{*}$ & $107 \pm 21$ & $122 \pm 48$ \\
\hline TA NH (mVs) & $0.012 \pm 0.004$ & $0.002 \pm 0.001$ & $0.002 \pm 0.001$ & $0.003 \pm 0.004$ \\
\hline $\mathrm{SH}(\%)$ & $154 \pm 72$ & $108 \pm 17$ & $104 \pm 27 \approx$ & $115 \pm 90^{*}$ \\
\hline LH (\%) & $96 \pm 14 \approx$ & $100 \pm 13 \approx$ & $101 \pm 14^{*}$ & $94 \pm 27=$ \\
\hline BF NH (mVs) & $0.011 \pm 0.006$ & $0.004 \pm 0.001$ & $0.004 \pm 0.002$ & $0.003 \pm 0.002$ \\
\hline SH (\&) & $67 \pm 35$ & $92 \pm 32=$ & $87 \pm 43=$ & $91 \pm 66 \approx$ \\
\hline LH (\%) & $107 \pm 26$ & $95 \pm 15 \approx$ & $105 \pm 25 \approx$ & $99 \pm 26 \approx$ \\
\hline VL. NH ( $m V s)$ & $0.012 \pm 0.005$ & $0.011 \pm 0.006$ & $0.006 \pm 0.003$ & $0.004 \pm 0.002$ \\
\hline SH (\%) & $84 \pm 41=$ & $82 \pm 36$ & $154 \pm 60$ & $142 \pm 71$ \\
\hline $\mathrm{LH}(\%)$ & $98 \pm 36 \approx$ & $98 \pm 8 \approx$ & $102 \pm 45$ & $114 \pm 66$ \\
\hline RF NH (mVs) & $0.008 \pm 0.004$ & $0.009 \pm 0.003$ & $0.004 \pm 0.002$ & $0.003 \pm 0.002$ \\
\hline SH (\%) & $110 \pm 57 \approx$ & $74 \pm 33$ & $168 \pm 69$ & $157 \pm 68$ \\
\hline LH (X) & $97 \pm 34$ & $99 \pm 17 \approx$ & $99 \pm 40 \approx$ & $117 \pm 61$ \\
\hline
\end{tabular}

lower. The differences between $\mathrm{NH}$ and $\mathrm{LH}$ were well below those observed between $\mathrm{NH}$ and $\mathrm{SH}$, but not always statistically insignificant: the peak forces of the $\mathrm{LH}$ compared to the $\mathrm{NH}$ were $102 \pm 4 \% \approx$, the RFD amounted to $96 \pm 6 \% \approx$, but the GCT was longer with $107 \pm 5 \%$.

\subsection{Electromyographic activity}

The EMG activity of the six leg muscles during four phases (PRE, SLR, MLR and LLR) is shown in Table 2. Again, absolute values apply to $\mathrm{NH}$ and relative values to $\mathrm{SH}$ and $\mathrm{LH}$. During the PRE phase, the EMG activity of the SH compared to the NH was lower for the majority of the recorded muscles. The SLR showed only small differences, whereas for the MLR and LLR the values of the SH were higher (with the exception of the knee and ankle flexors, i.e. TA and BF). The EMG activity of the LH compared to the NH for the phases PRE and SLR was statistically equal, but for the MLR and most notably the LLR the differences were not insignificant for all muscles.

\subsection{Motion analysis}

The absolute kinematic values of the $\mathrm{NH}$ as well as the differences between NH and SH are presented in Table 3 and illustrated in Fig. 4. The most important parameter (the ROM of the three joints) showed only small differences which were statistically insignificant. The leg stiffness during $\mathrm{NH}$ was $29 \pm 10 \mathrm{~N} / \mathrm{mm}$, whereas during $\mathrm{SH}$ it attained $79 \pm 10 \%$ of that value. 
Table 3

First row: Averaged values of all participants of the normal hoppings (NH). A negative value at the time of ground contact (GC) indicates that the joint was more flexed than during upright stance, a positive value indicates a more extended joint. Second row: averaged differences of all participants between sledge hoppings and normal hoppings in the three joint angles at $G C$ and the range of motion (ROM) from ground contact until takeoff. A negative value at GC indicates that the joint was more flexed during the SH than during the NH, a positive value indicates a more extended joint. For example, the value -5 for the knee angle at $G C$ means that the knee was flexed on average $5^{\circ}$ more during the SH. A negative value for the ROM indicates that the ROM was smaller for the SH.

\begin{tabular}{|c|c|c|c|c|c|c|c|}
\hline & GC ankle angle & GC knee angle & GC hip angle & Ankle ROM & Knee ROM & Hip ROM & Stiffness \\
\hline $\begin{array}{l}\mathrm{NH} \\
\Delta\end{array}$ & $\begin{array}{c}20 \pm 4^{\circ} \\
0 \pm 5^{n}=\end{array}$ & $\begin{array}{l}-13 \pm 8^{\circ} \\
-5 \pm 5^{n}\end{array}$ & $\begin{array}{r}-10 \pm 7^{\circ} \\
-9 \pm 6^{\circ}\end{array}$ & $\begin{array}{l}33 \pm 6^{\circ} \\
-1 \pm 7^{\circ}\end{array}$ & $\begin{array}{l}19 \pm 7^{\circ} \\
1 \pm 9^{\circ} \approx\end{array}$ & $\begin{array}{l}11 \pm 4^{\circ} \\
-2 \pm 4^{\circ}\end{array}$ & $\begin{array}{l}29 \pm 10 \mathrm{~N} / \mathrm{mm} \\
79 \pm 10 \%\end{array}$ \\
\hline
\end{tabular}

\section{Discussion}

The results show that the new system (characterized by a very light sledge and an attachment system that barely restricts the subject's movements) largely reduced the differences between sledge jumps and normal jumps that have been observed with previous SJSs (Bubeck and Gollhofer, 2001). For example, for jumps in the SJS used by Bubeck and Gollhofer (2001) the GCT was twice as high as for normal jumps, and the peak forces and the muscle preactivity were only half as high. The SJS used in the present study however reduced those differences to only one fifth and some of the recorded parameters were even statistically equal for the two types of hoppings. The only parameter that appears to differ to a higher extent is the EMG activity of the MLR and the LLR in some recorded muscles: for instance the LLR-value for GM was three times as high for the SH compared to the $\mathrm{NH}$ (see Table 2). However, this seemingly big difference is mainly due to the longer GCT in the SJS, since the EMG activity in the late phases of the SH is still high, whereas the EMG activity of the NH has already returned to a low base level (see Fig. 3).

The differences that do exist could be due to several factors. One reason could be the different position during the jumps (upright vs. supine), because even though the forces in the movement direction were matched, the force of gravity acted perpendicular to the movement direction in the SJS. This could explain at least part of the kinematic differences, e.g. the joint angles at GC. Moreover, the body position could have influenced the neural control (motor program) of the hoppings. For example, altered afferent feedback from cervical muscles might have changed the neural control of the muscular activity in the SJS (Gdowski et al., 2000; Manzoni et al., 1979; Noda, 1991).

Another possible reason for some of the observed differences could be the participants' lacking familiarity with the new device, resulting in a - consciously or unconsciously - cautious approach, i.e. a movement pattern with reduced stiffness (and consequently lower peak forces and longer $\mathrm{GCT}$ ) as a risk- and injuryprevention strategy. Further longitudinal studies with an adequate amount of training in the SJS could elucidate this possibility.

To test the possibility that the weight of the sledge (although very light compared to previous SJS) caused some differences, the hoppings with an additional weight equal to the sledge weight (LH) were incorporated. The results of that part of the study indicate that additional weight (in the form of a $5 \mathrm{~kg}$-weight vest) influenced most of the recorded parameters only to a small extent. However, it could explain some part of the observed differences between $\mathrm{NH}$ and $\mathrm{SH}$, for instance the $\mathrm{CCT}$ of the $\mathrm{LH}$ were $7 \%$ longer than during $\mathrm{NH}$. which is a third of the difference between SH and $\mathrm{NH}$ (22\%). Furthermore, the EMG activity of the LH during the phases of the MLR and LLR was elevated when compared to the $\mathrm{NH}$, just like it was the case for the SH. These results are consistent with the results of previous studies investigating the effect of additional load on the neuromuscular control in jump exercises. Gollhofer and Kyröläinen (1991) for example examined the effect of additional loads of 10 and $20 \mathrm{~kg}$ on drop jumps and reported a decreased preactivity of the recorded muscles, reduced GRF, reduced vertical stiffness and longer GCT. The authors attributed these effects to the reduced preactivity, which was interpreted as a method of stiffness control "in order to protect the tendomuscular system from high impact loads" (Gollhofer and Kyröläinen, 1991). The injury-prevention strategy of the neuromuscular system is probably the main reason not only for the huge differences between normal jumps and jumps in previous SJS (due to the high mass of the systems' moving parts, $\sim 70 \mathrm{~kg}$ in case of the SJS used by Bubeck and Gollhofer 2001), but also for the rather small differences observed in the present study. However, in the present study, the utilization of the injury-prevention strategy is possibly less due to the additional weight (as indicated by results of the LH part of the study) and more due to the fact that the main part of the force in the SJS was applied to the participant via the shoulders, exposing the spinal column to an unaccustomed load.

In conclusion, the present study clearly showed that hoppings in the new SIS were very similar to normal hoppings, with some parameters being even statistically equal. All requirements (preactivated muscles, short ground contact times, no delay between stretch and shortening, high leg stiffness) for jumps using the SSC are met. Therefore, the SJS can be used as a tool to investigate various characteristics of jumps under controlled conditions and with varying load, which facilitates the investigation of issues like energy storage, properties of the MTU or efficiency during jumps.

\section{Conflict of interest statement}

For the study with the title "A new sledge jump system that allows almost natural reactive jumps", the authors did not have any financial and personal relationships with other people or organisations that could inappropriately influence the study. The funding listed in the acknowledgements section was provided without any expectations concerning the results of the study.

\section{Acknowledgements}

The authors would like to thank Hans Schießl and Rainer Rawer of Novotec medical GmbH (as well as their coworkers) for building the sledge jump system. This study was supported in part by the European Space Agency (ESA Life Sciences Program TEC-MMG/2006/82).

\section{References}

Asmussen, E., Bonde-Petersen, F., 1974. Storage of elastic energy in skeletal muscles in man. Acta Physiologica Scandinavica 91, 385-392.

Borman, P.J., Chatfield, M.J., Damjanov, I., Jackson, P., 2009. Design and analysis of method equivalence studies. Analytical Chemistry $81,9849-9857$.

Bosco, C., Tarkka, L., Komi, P.V., 1982. Effect of elastic energy and myoelectrical potentiation of triceps surae during stretch-shortening cycle exercise. International Journal of Sports Medicine 3, 137-140. 
Bubeck D. 2002. Belastungsvariation und funktionelle Anpassungen im Dehnungs-Verkürzungs-Zyklus. Univ. Diss. Stuttgart, Universitäts-Bibliothek Stuttgart.

Bubeck, D. Gollhofer, A., 2001. Load induced changes of jump performance and activation patterns in free drop jump exercises and sledge jumps. European Journal of Sport Science 1, 1-17.

Finni. T., Ikegawa, S., Komi, P.V., 2001. Concentric force enhancement during human movement. Acta Physiologica Scandinavica 173, 369-377.

Fukashiro, S., Hay, D.C., Nagano. A., 2006. Biomechanical behavior of muscletendon complex during dynamic human movements. Journal of Applied Biomechanics 22, 131-147.

Gdowski, G.T., Boyle, R, McCrea, R.A., 2000. Sensory processing in the vestibular nuclei during active head movements. Archives Italiennes de Biologie 138, 15-28.

Gollhofer, A., Kyröläinen, H., 1991. Neuromuscular control of the human leg extensor muscles in jump exercises under various stretch-load conditions. International Journal of Sports Medicine 12,34-40.

Gollhofer, A, 1987. Komponenten der Schnellkraftleistungen im DehnungsVerkürzungs-Zyklus. SFT-Verlag, Erlensee.

Günther, M., Blickhan, R., 2002. Joint stiffness of the ankle and the knee in running. Journal of Biomechanics 35, 1459-1474.

Henchoz, Y., Malatesta, D., Gremion, G., Belli, A., 2006. Effects of the transition time between muscle-tendon stretch and shortening on mechanical efficiency. European Journal of Applied Physjology 96, 665-671.

Horita, T., Komi, P.V., Hämäläinen, l., Avela, J., 2003. Exhausting stretch-shortening cycle (SSC) exercise causes greater impairment in SSC performance than in pure concentric performance. European Journal of Applied Physiology $88,527-534$.
Komi, P.V., 1984. Physiological and biomechanical correlates of muscle function: effects of muscle structure and stretch-shortening cycle on force and speed Exercise and Sport Sciences Reviews 12, 81-121.

Komi, P.V., Gollhofer, A., 1997. Stretch-reflexes can have important role in force enhancement during SSC exercise. Journal of Applied Biomechanics. $451-460$.

Komi, P.V., Kaneko, M. Aura, O, 1987. EMG activity of the leg extensor muscles with special reference to mechanical efficiency in concentric and eccentric exercise. International Journal of Sports Medicine 8 (1), 22-29.

Kyröläinen, H., Komi. P.V., 1995. Differences in mechanical efficiency between power- and endurance-trained athletes while jumping. European Journal of Applied Physiology and Occupational Physiology 70, 36-44.

Lee, R.G., Tatton, W.G., 1978. Longloop reflexes in man: Clinical applications. In: Desmedt, J.E. (Ed.), Cerebral Motor Control in Man: Long Loop Mechanisms. Karger, Basel, pp. 320-333.

Manzoni, D., Pompeiano, O., Stampacchia, G., 1979. Cervical control of posture and movements. Brain Research 169, 615-619.

Marsden, C.D. Merton, P.A., Morton, H.B., Adam, J. Hallett, M, 1978. Automatic and voluntary responses to muscle stretch in man. In: Desmedt, J.E. (Ed.) Cerebral Motor control in Man: Long Loop Mechanisms. Karger. Basel, pp. $167-177$.

Noda, $T ., 1991$. The importance of cervical muscles in responses of galvanic body sway test. Nippon Jibiinkoka Gakkai Kaiho 94, 1098-1103.

Sinkjaer, T., Andersen, J.B., Nielsen, J.F., Hansen, H.]., 1999. Soleus long-latency stretch reflexes during walking in healthy and spastic humans. Clinical Neurophysiology : Official Journal of the International Federation of Clinical Neurophysiology 110, 951-959. 